\title{
Reduced ex vivo susceptibility of Plasmodium falciparum after oral artemether- lumefantrine treatment in Mali
}

\author{
Souleymane Dama, Hamidou Niangaly, Amed Ouattara, Issaka Sagara, Sekou Sissoko, Oumar Bila Traore, \\ Amadou Bamadio, Niawanlou Dara, Moussa Djimde, Mohamed Lamine Alhousseini, Siaka Goita, \\ Hamma Maiga, Antoine Dara, Ogobara K. Doumbo and Abdoulaye A. Djimde*
}

\begin{abstract}
Background: Artemisinin-based combination therapy is the recommended first-line treatment for uncomplicated falciparum malaria worldwide. However, recent studies conducted in Mali showed an increased frequency of recurrent parasitaemia following artemether-lumefantrine $(\mathrm{AL})$ treatment.

Methods: Study samples were collected during a large WANECAM study. Ex-vivo Plasmodium falciparum sensitivity to artemether and lumefantrine was assessed using the tritiated hypoxanthine-based assay. The prevalence of molecular markers of anti-malarial drug resistance (pfcrt K76T, pfmdr1 N86Y and K13-propeller) were measured by PCR and/or sequencing.

Results: Overall 61 samples were successfully analysed in ex vivo studies. Mean $I_{50}$ increased significantly between baseline and recurrent parasites for both artemether (1.6 nM vs $3.2 \mathrm{nM}, \mathrm{p}<0.001$ ) and lumefantrine (1.4 nM vs $3.4 \mathrm{nM}$, $\mathrm{p}=0.004)$. Wild type Pfmdr1 N86 allele was selected after treatment (71 vs 91\%, 112 of 158 vs 95 of 105, p < 0.001) but not the wild type pfcrt K76 variant (23.5 vs 24.8\%, 40 of 170 vs 26 of 105, $p=0.9$ ). Three non-synonymous K13-propeller SNPs (A522C, A578S, and G638R) were found with allele frequencies $<2 \%$.
\end{abstract}

Conclusion: Malian post-AL P. falciparum isolates were less susceptible to artemether and lumefantrine than baseline isolates.

Keywords: Artemether, Lumefantrine, Plasmodium, Ex vivo, In vivo, K13 propeller

\section{Background}

Artemisinin-based combination therapy (ACT) is currently the first-line treatment for uncomplicated falciparum malaria worldwide, in both the public and private sectors $[1,2]$. Lumefantrine has never been used alone in people [3]. Studies of in vitro susceptibility of Plasmodium falciparum to lumefantrine from West African countries showed $\mathrm{IC}_{50}$ ten times below the resistance threshold of $150 \mathrm{nM}$ [4-7], while P. falciparum isolates

\footnotetext{
*Correspondence: adjimde@icermali.org

Malaria Research and Training Center, Faculty of Pharmacy and Faculty

of Medicine and Dentistry, University of Sciences, Technique

and Technology of Bamako, P.O. Box 1805, Bamako, Mali
}

from South-East Asia show higher mean $\mathrm{IC}_{50}$ that are closed to that threshold [8].

Several studies have investigated the role of $p f m d r 1$ gene in lumefantrine tolerance. Sisowath et al. $[9,10]$ and Baliraine et al. [11] reported that lumefantrine pressure provides the main selective force on the pfmdr 1 N86 allele and leads to resistance to lumefantrine. Happi et al. [12] reported that the pfmdr1_N86_F184_D1246 (NFD) haplotype rose from $10 \%$ in baseline samples to $75 \%$ in breakthrough parasites, an indication of its enhanced survival fitness. Similar observations were reported in three different studies in East Africa [11, 13, 14]. Furthermore, an association between lumefantrine $\mathrm{IC}_{50}$ and pfmdr1 gene polymorphism was demonstrated in Thai isolates [15]. 
Lumefantrine was shown to have synergistic interaction with artemisinin derivatives in vitro in continuous culture [16]. Hence, artemether was the first artemisinin derivative to be combined in fixed dose with a partner drug as artemether-lumefantrine. Delayed parasite clearance is the phenotype of in vivo resistance to artemisinin and its derivatives in the field [17]. Witkowski et al. [18] reported that increased tolerance to artemisinin in vitro was seen in quiescent $P$. falciparum parasite from Thailand-Cambodia border. Noedl et al. [19] reported the first cases of artemisinin resistance in vitro in Cambodia.

A reduced susceptibility of $P$. falciparum to artemisinin was not detected in mature forms but in young ring stages [20]. The in vitro and ex vivo ring stage survival assay (RSA) on $0-3 \mathrm{~h}$ rings $\left(\mathrm{RSA}_{0-3}\right)$ correlated best with in vivo artemisinin resistance as measured by delayed parasite clearance [21]. Flow cytometry-based ring-stage survival assay performs as well as light microscopy and can be used to standardize the collection of RSA data for P. falciparum artemisinin resistance between research groups in laboratory and field settings [22].

Recent studies have identified P. falciparum Kelch 13 ( $p f \mathrm{~K} 13)$ polymorphisms to be associated with delayed parasite clearance time after ACT in Southeast Asia [23]. Three key point mutations in the $p f \mathrm{~K} 13$ gene were associated with delayed clearance of $P$. falciparum following artemisinin treatment in Southeast Asia [24]. Most studies assessing the relationship between delayed parasite clearance time and $\mathrm{K} 13$ mutations have been conducted in Asia. The possibility that artemisinin resistance might also appear or introduced in sub-Saharan Africa necessitates careful surveillance through periodic in vivo and ex vivo efficacy studies in malaria endemic regions. Although several studies of the efficacy of artemetherlumefantrine in Africa showed high efficacy after molecular correction, the cure rate without PCR correction was lower [25-29]. Similarly, studies in Mali showed a significant number of recurrent parasitaemia following artemether-lumefantrine (AL) treatment [30, 31]. The study hypothesis was that these recurrent parasites are less sensitive to AL in ex vivo study.

\section{Methods}

\section{Sample collection}

Blood samples were collected from the patient enrolled in artemether-lumefantrine arm during the large ongoing network study [32]. Briefly $2-3 \mathrm{ml}$ of blood was obtained by venipuncture from patient with $P$. falciparum mono-infections at least 2250 trophozoites per $\mu \mathrm{l}(0.05 \%)$ or greater parasitaemia by using the EDTA Vacutainer ${ }^{\circledR}$ tubes. Day 0 samples were collected before treatment administration and independents Day recurrent parasites samples were collected after treatment initiation between
Day 28 and Day 42. Samples were transported by car from Sotuba and Kollé to the Culture Room of Malaria Research and Training Centre at $4-8{ }^{\circ} \mathrm{C}$ where ex vivo has been done. All samples were cultivated ex vivo immediately or for no longer than $24 \mathrm{~h}$.

\section{Drugs dilution and plate coating, preparation of culture media}

Before sample collection, two anti-malarial drugs (artemether A9361-25MG, SIGMA and lumefantrine L5420-25MG) were dissolved and distributed in appropriate solutions (methanol and ethanol). The starting solutions were prepared from the powder contained in glass vials. Artemether was dissolved at a concentration of $0.1 \mathrm{mg} / \mathrm{ml}$ with methanol. Lumefantrine was dissolved in ethanol to have a concentration of $0.01 \mathrm{mg} / \mathrm{ml}$. These starting solutions have been used directly or stored in freezer for the future use. Dilutions calculations were double checked before. All information about weight, solvent were recorded in laboratory book. The preparation of serial dilutions were made in sterile glass flask that allowed to have following concentrations (2.34-600 nM for artemether and 1.25-320 $\mathrm{nM}$ for lumefantrine). Each serial concentration have been made in triplicate in 96-well tissue culture plate and dried under a laminar flow hood. RPMI 1640 (R6504-10L) powder with L-glutamate was dissolved in cell grade water and supplemented with sodium bicarbonate and HEPES. After checking $\mathrm{pH}$, which should be 7.2 media was supplemented with $10 \%$ of human serum.

\section{Ex vivo sensitivity testing}

For ex vivo drug sensitivity testing, a semi-automated radioactive microplate method was used to measure the $\mathrm{IC}_{50}$ as described in a previous study [33]. Briefly, at day 0 , ring-form parasites obtained after blood collection were washed three times in RPMI 1640 media. Infected Red blood cells were suspended in $10 \%$ human serum completed culture media. After adjustment of parasitaemia between 0.5 and $1 \%$ and hematocrit at $1 \%$, an equivalent of $1 \mu \mathrm{Ci}$ per well of tritiated hypoxanthine with a specific activity of $14.1 \mathrm{Ci} / \mathrm{mmol}$ (Perkin-Elmer, Foster City, CA) was added in a parasite suspension. A final volume of $200 \mu \mathrm{l}$ per well of parasite suspension was placed into drug-prefilled 96-well tissue culture plates. The parasite suspension was mixed and incubated with each drug at various concentrations of drug at $37{ }^{\circ} \mathrm{C}$ with $5 \%$ $\mathrm{CO}_{2}, 5 \% \mathrm{O}_{2}$ and $90 \% \mathrm{~N}_{2}$ for $42 \mathrm{~h}$. After one step of freezing and thawing, the parasites were collected on filter plate, dried and $50 \mu \mathrm{l}$ of scintillation cocktail (Optiphase Supermix; Perkin-Elmer) was added on each well. Tritium incorporation was determined with a beta-counter (Wallac 1450 microbeta trilux; Perkin-Elmer), and the 
$\mathrm{IC}_{50}$ was determined after the drug concentration was plotted against the radioactivity by the online $\mathrm{IC}_{50}$ estimator [34]. Three wells without drug for each concentration panel and the reference clones 3D7 and Dd2 were used as control.

\section{Molecular analysis}

For the molecular analyses purpose dried blood spot samples (DBS) collected during the in vivo study [32]. To evaluate the prevalence of molecular markers of drug resistance and discriminate reinfection from recrudescence, DBS were used to extract parasite DNA using Qiagen mini kit (Qiagen, Valencia, CA) according to the manufacturer's recommendations.

Plasmodium falciparum chloroquine-resistance transporter ( $p f c r t) \mathrm{K} 76 \mathrm{~T}$ and $P$. falciparum multidrug resistance 1 (pfmdr1) N86Y mutations were genotyped in pre-treatment samples and in parasitaemia occurring in recurrent follow-up day [35]. A nested PCR strategy based on previously published protocols [24, 36] was used to amplify a final 850 bp of the K13-propeller domain product (for PCR conditions see Ref. [36]). PCR products were sent to Macrogen Europe for sequencing. $p f \mathrm{~K} 13$ sequence editing and analysis were conducted in Bamako, Mali.

\section{Statistical analysis}

$\mathrm{IC}_{50}$ values were analysed after logarithmic transformation and expressed as the geometric mean of the $\mathrm{IC}_{50}$ and the confidence interval 95\% (95\% CI). These continuous variables were compared using the independent Student's $t$ test. Prevalence of molecular markers of drug resistance was compared using the Chi Square test. All statistical analyses were done with Stata version 12.0 (Stata Corp., College Station, TX).

\section{Results}

Ex vivo efficacy

Full in vivo efficacy results for this study were reported elsewhere [32]. Overall 61 samples were successfully analysed in this ex vivo study. 38 samples were collected on day 0 before treatment initiation. Twenty-three samples were collected from recurrent parasites between day 28 to day 42. All samples included in this study had parasitaemia at least $0.05 \%$ or greater parasitaemia. $20 \%$ of ex vivo samples came from Kollé and 80\% came from Sotuba. The geometric mean difference between pretreatment and post-treatment $\mathrm{IC}_{50}$ were $1.6 \mathrm{nM}(95 \%$ CI, 1.4-1.9) vs $3.2 \mathrm{nM}$ (95\% CI, 2.5-4.2) for artemether $(\mathrm{p}<0.01)$ and $1.4 \mathrm{nM}(95 \% \mathrm{CI}, 1.2-2.7)$ vs $3.4 \mathrm{nM}(95 \%$ $\mathrm{CI}, 2.0-5.9)$ for lumefantrine $(\mathrm{p}=0.004)$ (Table 1$)$.

\section{PCR-RFLP analyses of pfcrt K76T and pfmdr1 N86Y single nucleotide polymorphisms}

Overall 275 samples were included for the molecular analysis. From which 170 samples were sample available at baseline and 105 were available at the follow-up day. $45 \%$ of the sample came from Kolle and $55 \%$ came from Sotuba. Prevalence of $p f m d r 1$ and $p f c r t$ alleles were compared between pre-treatment $P$. falciparum parasite isolates and parasites that emerged after treatment. At baseline pfcrt $\mathrm{K} 76$ was detected in $23.5 \%$ (40/170) while pfmdr1 N86 was detected in 71\% (112/158) of P. falciparum isolates. After treatment with artemether-lumefantrine the prevalence of $p f c r t \mathrm{~K} 76$ and $p f m d r 1$ N86 were $24.8 \%(26 / 105)$ and $91 \%(95 / 105)$, respectively $(\mathrm{p}=0.9$ for $p f c r t$ and $\mathrm{p}<0.001$ for $p f m d r 1$ ) (Fig. 1). There was no statistically significant association between $p f c r t$ K76 or pfmdr1 N86 and P. falciparum ex vivo responses $(\mathrm{p}>0.05)$.

\section{Kelch13-propeller domain analysis}

The K13-propeller domain sequences were generated from 192 samples collected in both Kolle and Sotuba (Genbank accession numbers AD1-AD167). Twenty-five samples had poor quality sequences and were excluded from this analysis. Using 3D7 as the reference strain, three low frequency non-synonymous SNPs were identified at codons A522C (1.2\%), A578S (0.6\%), and G638R $(0.6 \%)$ in the K13-propeller domain. Three alleles were observed in Sotuba (3D7, A578S, G638R alleles) and two in Kolle (3D7, S522C allele).

\section{Discussion}

The finding of this study show that the ex vivo efficacy of both artemether and lumefantrine was significantly reduced in post-AL parasites compared to pre-treatment parasites even though the respective $\mathrm{IC}_{50}$ were all within the respective sensitive ranges of each drug [37]. The geometric mean $\mathrm{IC}_{50}$ values of the artemether measured

Table 1 Geometric mean $I C_{50}$ before and after treatment for artemether and lumefantrine

\begin{tabular}{lllr}
\hline $\mathbf{I} \mathbf{5}_{\mathbf{5 0}}$ drugs & $\begin{array}{l}\text { Pre-treatment geometric mean } \\
\left.\mathbf{( 9 5 \%}{ }^{\mathrm{a}} \mathbf{C l}\right)\end{array}$ & $\begin{array}{l}\text { Post-treatment geometric mean } \\
\mathbf{( 9 5 \% ~ C l )}\end{array}$ & $\mathbf{p ~ v a l u e s}$ \\
\hline Artemether & $1.6(1.4-1.9)$ & $3.2(2.5-4.2)$ & $<0.001$ \\
Lumefantrine & $1.4(1.2-1.7)$ & $3.4(2.0-5.9)$ & 0.004 \\
\hline
\end{tabular}

${ }^{a}$ Confidence interval 


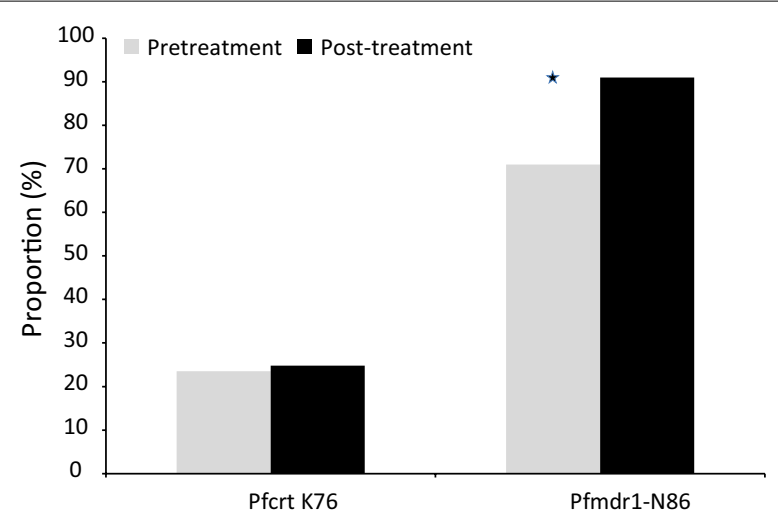

Fig. 1 PfcrtK76 and Pfmdr1-N86 frequency between pretreatment versus post-treatment parasites $\left({ }^{*} \mathrm{p}<0.05\right)$

in the present study are similar to published data from studies conducted in other Africans countries [7, 38, 39]. Artemether mean $\mathrm{IC}_{50}$ was six times lower than the $21.2 \mathrm{nM}$ reported in a study conducted in South-Est Asia in 2011 [8]. Lumefantrine mean $\mathrm{IC}_{50}$ was lower than that reported in a previous study conducted in Mali [6] and 4 times lower than the $15.1 \mathrm{nM}$ reported in North-Western Thailand [40].

Although there was an increase in $\mathrm{IC}_{50}$ in post-AL parasites, in vivo study conducted at the same period of our sample collection found that the corrected clinical efficacy of oral artemether-lumefantrine remained high for the treatment of uncomplicated malaria [32]. All the samples tested ex vivo had an adequate clinical and parasitological response after molecular correction. These results were comparable to previous reports in others sites in Mali [30, 41, 42]. Similar finding was reported in artesunate monotherapy study conducted in Mali in 2010 [25]. The cure rate was also similar to the $99 \%$ efficacy reported in a Kenyan study in 2010 [43]. These findings confirm that AL is still efficacious on malaria in Mali.

This study confirms previous findings of a selection of pfmdr1 N86 following artemether-lumefantrine treatment $[9-11,44]$. These molecular findings lend further support to the increased $\mathrm{IC}_{50}$ found for lumefantrine as pfmdr1 N86 was shown to be associated with P. falciparum in vitro resistance to lumefantrine [45]. The prevalence of $p f c r t \mathrm{~K} 76$ allele remained stable after treatment with artemether-lumefantrine in this cohort study as reported in recent studies conducted in other Africans countries [46, 47]. The lack of difference of $p f c r t$ wild type allele in this dataset between the days of failure compared to the baseline may probably be explained by the high prevalence of the mutant allele at baseline.

The prevalence of K13-propeller mutations was low in Malian parasite population. In vivo artemisinin resistance and delayed parasite clearance due to artemisinin resistance have not been reported in Mali [44]. None of the mutations associated with prolonged parasite clearance time after artemisinin-based drugs reported in Cambodia and other Southeast Asian countries were observed in Mali [24]. Of the three non-synonymous SNPs identified in this study only A578S was reported in previous studies in Mali [36, 48]; A522C and G638R have not yet been previously reported. All these SNPs were observed in the pre-treatment samples while no SNPs were observed in post-treatment samples.

The demonstrated continued efficacy of AL in this study has major implications for malaria control in West Africa. Indeed, AL is a centerpiece for malaria casemanagement in the Sahel belt where current deployment of Seasonal Malaria Chemoprevention (SMC) with sulfadoxine-pyrimethamine (SP) plus amodiaquine (AQ) discourages the use of $\mathrm{AQ}$ containing ACTs. In addition, because AL selects the wild type allele $p f m d r 1 \mathrm{~N} 86$, which is associated with increased susceptibility to chloroquine and AQ [49], the use of AL in malaria case management in West Africa would prolong the effectiveness of SPAQ for SMC.

Because of logistical constraints complete paired sample set were not obtained for most patients included in the ex vivo assays while paired sample are ideally the best to do before and after. For these reasons independent Student's t test was used to compare group before and after treatment.

\section{Conclusion}

Although AL is efficacious, ex vivo $P$. falciparum decreased susceptibility and selection of pfmdr1 86Y indicate the need not only for aggressive monitoring of parasite response to this mainstream ACT but also for the introduction of new types of ACT in this sub-region.

\section{Authors' contributions}

SD contributed to the design of the study, to patient recruitment and followup, conducted the molecular and ex vivo analyses and wrote the first draft of the manuscript. HN contributed to data management and statistical analysis. IS contributed to the design of the study, supervised the clinical study and assisted with data interpretation. SS contributed to the molecular and ex vivo analyses. $\mathrm{OA}$ and $\mathrm{AD}$ did the analysis of the K13-propeller polymorphism. $\mathrm{OBT}, \mathrm{AB}, \mathrm{MD}, \mathrm{HM}, \mathrm{ND}, \mathrm{SG}$ and MLA collected in vivo data. OKD contributed to the supervision of the study and manuscript preparation. AAD contributed to the design of the study, supervised the clinical study, assisted with data interpretation and manuscript preparation. All authors read and approved the final manuscript.

\section{Acknowledgements}

We would like to acknowledge the collaboration of the study population and local staff.

\section{Competing interests}

The authors declare that they have no competing interests. 


\section{Availability of data and materials}

The datasets during and/or analysed during the current study available from the corresponding author on reasonable request.

\section{Ethics approval and consent to participate}

The Ethics Committee of the Faculty of Medicine, Pharmacy, and OdontoStomatology, University of Sciences, Techniques and Technology of Bamako approved the protocol to the number ( $\left.{ }^{\circ} 10 \_79 \_/ F M P O S\right)$. Each patient (or their parent or guardian) gave fully informed written consent prior to enrollment.

\section{Funding}

This study was funded by the European and Developing Countries Clinical Trials Partnership (EDCTP IP_07_31060_002), West African Network for Clinical Trials of Antimalarial Drugs (WANECAM) and Medicines for Malaria Venture (MMV). SD was supported by a Wellcome Trust funded Ph.D. Scholarship through the Institute of Infectious Diseases of Poverty (IIDP, Award \#087536/Z/08/Z).

Received: 8 September 2016 Accepted: 18 January 2017 Published online: 02 February 2017

\section{References}

1. WHO. Guidelines for the treatment of malaria. 2nd ed. Geneva: World Health Organization; 2010

2. WHO. Global Malaria Programme. World Malaria Report 2014. Geneva: World Health Organization; 2014.

3. WHO. Guidelines for the treatment of malaria. 3rd ed. Geneva: World Health Organization; 2015.

4. Basco LK, Bickii J, Ringwald P. In vitro activity of lumefantrine (benflumetol) against clinical isolates of Plasmodium falciparum in Yaoundé, Cameroon. Antimicrob Agents Chemother. 1998;42:2347-51.

5. Pradines B, Tall A, Fusai T, Spiegel A, Hienne R, Rogier C, et al. In vitro activities of benflumetol against 158 Senegalese isolates of Plasmodium falciparum in comparison with those of standard antimalarial drugs. Antimicrob Agents Chemother. 1999;43:418-20.

6. Kaddouri H, Djimdé A, Dama S, Kodio A, Tekete M, Hubert V, et al. Baseline in vitro efficacy of ACT component drugs on Plasmodium falciparum clinical isolates from Mali. Int J Parasitol. 2008;38:791-8.

7. Quashie NB, Duah NO, Abuaku B, Quaye L, Ayanful-Torgby R, Akwoviah GA, et al. A SYBR Green 1-based in vitro test of susceptibility of Ghanaian Plasmodium falciparum clinical isolates to a panel of anti-malarial drugs. Malar J. 2013;12:450.

8. Pradines B, Bertaux L, Pomares C, Delaunay P, Marty P. Reduced in vitro susceptibility to artemisinin derivatives associated with multi-resistance in a traveller returning from South-East Asia. Malar J. 2011:10:268.

9. Sisowath C, Strömberg J, Mårtensson A, Msellem M, Obondo C, Björkman $\mathrm{A}$, et al. In vivo selection of Plasmodium falciparum pfmdr $186 \mathrm{~N}$ coding alleles by artemether-lumefantrine (Coartem). J Infect Dis. 2005;191:1014-7.

10. Sisowath C, Ferreira PE, Bustamante LY, Dahlström S, Mårtensson A, Björkman $A$, et al. The role of pfmdr1 in Plasmodium falciparum tolerance to artemether-lumefantrine in Africa. Trop Med Int Health. 2007;12:736-42.

11. Baliraine FN, Rosenthal PJ. Prolonged selection of pfmdr1 polymorphisms after treatment of falciparum malaria with artemether-lumefantrine in Uganda. J Infect Dis. 2011;204:1120-4.

12. Happi CT, Gbotosho GO, Folarin OA, Sowunmi A, Hudson T, O'Neil M, et al. Selection of Plasmodium falciparum multidrug resistance gene 1 alleles in asexual stages and gametocytes by artemether-lumefantrine in Nigerian children with uncomplicated falciparum malaria. Antimicrob Agents Chemother. 2009;53:888-95.

13. Humphreys GS, Merinopoulos I, Ahmed J, Whitty CJM, Mutabingwa TK, Sutherland CJ, et al. Amodiaquine and artemether-lumefantrine select distinct alleles of the Plasmodium falciparum mdr 1 gene in Tanzanian children treated for uncomplicated malaria. Antimicrob Agents Chemother. 2007:51:991-7.

14. Gadalla NB, Adam I, Elzaki SE, Bashir S, Mukhtar I, Oguike M, et al. Increased pfmdr 1 copy number and sequence polymorphisms in
Plasmodium falciparum isolates from Sudanese malaria patients treated with artemether-lumefantrine. Antimicrob Agents Chemother. 2011:55:5408-11.

15. Mungthin M, Khositnithikul R, Sitthichot N, Suwandittakul N, Wattanaveeradej $\mathrm{V}$, Ward SA, et al. Association between the pfmdr1 gene and in vitro artemether and lumefantrine sensitivity in thai isolates of Plasmodium falciparum. Am J Trop Med Hyg. 2010;83:1005-9.

16. Hassan Alin M, Björkman A, Wernsdorfer WH. Synergism of benflumetol and artemether in Plasmodium falciparum. Am J Trop Med Hyg. 1999;61:439-45.

17. Dondorp AM, Nosten F, Yi P, Das D, Hanpithakpong W, Lee SJ, et al. Artemisinin resistance in Plasmodium falciparum malaria. N Engl J Med. 2012;361:455-67.

18. Witkowski B, Lelièvre J, Barragán MJ, Laurent V, Su XZ, Berry A, et al. Increased tolerance to artemisinin in Plasmodium falciparum is mediated by a quiescence mechanism. Antimicrob Agents Chemother. 2010;54:1872-7.

19. Noedl H, Se Y, Schaecher K, Smith BL, Socheat D, Fukuda MM, et al. Evidence of artemisinin-resistant malaria in western Cambodia. N Engl J Med. 2008:359:2619-20.

20. Witkowski B, Khim N, Chim P, Kim S, Ke S, Kloeung N, et al. Reduced artemisinin susceptibility of Plasmodium falciparum ring stages in Western Cambodia. Antimicrob Agents Chemother. 2013;57:914-23.

21. Witkowski B, Amaratunga C, Khim N, Sreng S, Chim P, Kim S, et al. Novel phenotypic assays for the detection of artemisinin-resistant Plasmodium falciparum malaria in Cambodia: in vitro and ex vivo drug-response studies. Lancet Infect Dis. 2013:13:1043-9.

22. Amaratunga C, Neal AT, Fairhurst RM. Flow cytometry-based analysis of artemisinin-resistant Plasmodium falciparum in the ring-stage survival assay. Antimicrob Agents Chemother. 2014;58:4938-40.

23. Takala-Harrison S, Clark TG, Jacob CG, Cummings MP, Miotto O, Dondorp AM, et al. Genetic loci associated with delayed clearance of Plasmodium falciparum following artemisinin treatment in Southeast Asia. Proc Nat Acad Sci USA. 2013;110:240-5.

24. Ariey F, Witkowski B, Amaratunga C, Beghain J, Langlois A-C, Khim N, et al. A molecular marker of artemisinin-resistant Plasmodium falciparum malaria. Nature. 2014;505:50-5.

25. Abdulla S, Sagara I, Borrmann S, D'Alessandro U, González R, Hamel M, et al. Efficacy and safety of artemether-lumefantrine dispersible tablets compared with crushed commercial tablets in African infants and children with uncomplicated malaria: a randomised, single-blind, multicentre trial. Lancet. 2008:372:1819-27.

26. Nhama A, Bassat Q, Enosse S, Nhacolo A, Mutemba R, Carvalho E, et al. In vivo efficacy of artemether-lumefantrine and artesunate-amodiaquine for the treatment of uncomplicated falciparum malaria in children: a multisite, open-label, two-cohort, clinical trial in Mozambique. Malar J. 2014;13:309.

27. Ndiaye JL, Randrianarivelojosia M, Sagara I, Brasseur P, Ndiaye I, Faye B, et al. Randomized, multicentre assessment of the efficacy and safety of ASAQ-a fixed-dose artesunate-amodiaquine combination therapy in the treatment of uncomplicated Plasmodium falciparum malaria. Malar J. 2009:8:125.

28. Chanda P, Hawela M, Kango M, Sipilanyambe N. Assessment of the therapeutic efficacy of a paediatric formulation of artemether-lumefantrine (Coartesiane) for the treatment of uncomplicated Plasmodium falciparum in children in Zambia. Malar J. 2006:5:75

29. van den Broek I, Kitz C, Al Attas S, Libama F, Balasegaram M, Guthmann J-P. Efficacy of three artemisinin combination therapies for the treatment of uncomplicated Plasmodium falciparum malaria in the Republic of Congo. Malar J. 2006;5:113.

30. Sagara I, Diallo A, Kone M, Coulibaly M, Diawara SI, Guindo O, et al. A randomized trial of artesunate-mefloquine versus artemether-lumefantrine for treatment of uncomplicated Plasmodium falciparum malaria in Mali. Am J Trop Med Hyg. 2008;79:655-61

31. Sagara I, Dicko A, Djimde A, Guindo O, Kone M, Tolo Y, et al. A randomized trial of artesunate-sulfamethoxypyrazine-pyrimethamine versus artemether-lumefantrine for the treatment of uncomplicated Plasmodium falciparum malaria in Mali. Am J Trop Med Hyg. 2006;75:630-6.

32. Sagara I, Beavogui AH, Zongo I, Soulama I, Borghini-Fuhrer I, Fofana B, et al. Safety and efficacy of re-treatments with pyronaridine-artesunate in African patients with malaria: a substudy of the WANECAM randomised trial. Lancet Infect Dis. 2015;16:189-98. 
33. Desjardins RE, Canfield CJ, Haynes JD, Chulay JD. Quantitative assessment of antimalarial activity in vitro by a semiautomated microdilution technique. Antimicrob Agents Chemother. 1979;16:710-8.

34. ICEstimator (version 1.2). http://www.antimalarialicestimator.net/ Methodintro.htm

35. Djimdé A, Doumbo OK, Cortese JF, Kayentao K, Doumbo S, Diourté Y, et al. A molecular marker for chloroquine-resistant falciparum malaria. N Engl J Med. 2001;344:257-63.

36. Ouattara A, Kone A, Adams M, Fofana B, Maiga AW, Hampton S, et al. Polymorphisms in the K13-propeller gene in artemisinin-susceptible Plasmodium falciparum parasites from Bougoula-Hameau and Bandiagara. Mali. Am J Trop Med Hyg. 2015;92:1202-6.

37. Ekland EH, Fidock DA. In vitro evaluations of antimalarial drugs and their relevance to clinical outcomes. Int J Parasitol. 2008;38:743-7.

38. Fall B, Diawara S, Sow K, Baret E, Diatta B, Fall KB, et al. Ex vivo susceptibility of Plasmodium falciparum isolates from Dakar, Senegal, to seven standard anti-malarial drugs. Malar J. 2011;10:310

39. Fall B, Camara C, Fall M, Nakoulima A, Dionne P, Diatta B, et al. Plasmodium falciparum susceptibility to standard and potential anti-malarial drugs in Dakar, Senegal, during the 2013-2014 malaria season. Malar J. 2015;14:60

40. Pilz JB, Wernsdorfer G, Sirichaisinthop J, Rojanawatsirivet C, Wiedermann G. Wernsdorfer WH. In vitro sensitivity of Plasmodium falciparum to lumefantrine in north-western Thailand. Wien Klin Wochenschr. 2004;116:41-6.

41. Kayentao K, Doumbo OK, Pénali LK, Offianan AT, Bhatt KM, Kimani J, et al. Pyronaridine-artesunate granules versus artemether-lumefantrine crushed tablets in children with Plasmodium falciparum malaria: a randomized controlled trial. Malar J. 2012;11:364.

42. Maiga AW, Fofana B, Sagara I, Dembele D, Dara A, Traore OB, et al. No evidence of delayed parasite clearance after oral artesunate treatment of uncomplicated falciparum malaria in Mali. Am J Trop Med Hyg. 2012;87:23-8.

43. Ogutu BR, Onyango KO, Koskei N, Omondi EK, Ongecha JM, Otieno GA, et al. Efficacy and safety of artemether-lumefantrine and dihydroartemisinin-piperaquine in the treatment of uncomplicated Plasmodium falciparum malaria in Kenyan children aged less than five years: results of an open-label, randomized, single-centre study. Malar J. 2014;13:33.
44. Baraka V, Tinto H, Valea I, Fitzhenry R, Delgado-Ratto C, Mbonye MK, et al. In vivo selection of Plasmodium falciparum Pfcrt and Pfmdr1 variants by artemether-lumefantrine and dihydroartemisinin-piperaquine in Burkina Faso. Antimicrob Agents Chemother. 2015;59:734-7.

45. Wurtz N, Fall B, Pascual A, Fall M, Baret E, Camara C, et al. Role of Pfmdr1 in in vitro Plasmodium falciparum susceptibility to chloroquine, quinine, monodesethylamodiaquine, mefloquine, lumefantrine, and dihydroartemisinin. Antimicrob Agents Chemother. 2014;58:7032-40

46. Lekana-Douki JB, Boutamba SDD, Zatra R, Edou SEZ, Ekomy H, Bisvigou $\mathrm{U}$, et al. Increased prevalence of the Plasmodium falciparum Pfmdr1 $86 \mathrm{~N}$ genotype among field isolates from Franceville, Gabon after replacement of chloroquine by artemether-lumefantrine and artesunate-mefloquine. Infect Genet Evol. 2011;11:512-7.

47. Duah NO, Matrevi SA, de Souza DK, Binnah DD, Tamakloe MM, Opoku VS, et al. Increased pfmdr1 gene copy number and the decline in pfcrt and pfmdr 1 resistance alleles in Ghanaian Plasmodium falciparum isolates after the change of anti-malarial drug treatment policy. Malar J. 2013:12:377.

48. Taylor SM, Parobek CM, De Conti DK, Kayentao K, Coulibaly SO, Greenwood BM, et al. Absence of putative artemisinin resistance mutations among Plasmodium falciparum in sub-Saharan Africa: a molecular epidemiologic study. J Infect Dis. 2015;211:680-8.

49. Venkatesan M, Gadalla NB, Stepniewska K, Dahal P, Nsanzabana C, Moriera C, et al. Polymorphisms in Plasmodium falciparum chloroquine resistance transporter and multidrug resistance 1 genes: parasite risk factors that affect treatment outcomes for P. falciparum malaria after artemether-lumefantrine and artesunate-amodiaquine. Am J Trop Med Hyg. 2014;91:833-43.

\section{Submit your next manuscript to BioMed Central and we will help you at every step:}

- We accept pre-submission inquiries

- Our selector tool helps you to find the most relevant journal

- We provide round the clock customer support

- Convenient online submission

- Thorough peer review

- Inclusion in PubMed and all major indexing services

- Maximum visibility for your research

Submit your manuscript at www.biomedcentral.com/submit
() Biomed Central 